\title{
Hsa_circ_0004831 serves as a blood-based prognostic biomarker for colorectal cancer and its potentially circRNA-miRNA-mRNA regulatory network construction
}

\author{
Linlin Xing ${ }^{1}\left(\mathbb{0}\right.$, Mengyan $X i a^{1}$, Xin Jiao ${ }^{1}$ and Ling Fan ${ }^{2 *}$
}

\begin{abstract}
Background: Colorectal cancer $(C R C)$ is a common malignant tumor with unsatisfactory overall prognosis. CircRNAs could be promising prognostic biomarkers in cancers, and play important role in the process of tumorigenesis and progression. Here, we explored the role of hsa_circ_0004831 in blood extracellular vesicles and its prognostic value in CRC.

Methods: The circRNA and mRNA expression level matrix in extracellular vesicles of CRC and normal samples were obtained from the exoRBase database. The corresponding miRNA expression level matrix in extracellular vesicles was downloaded from the BBCancer database. Differentially expressed circRNAs, miRNAs and mRNAs were identified using the limma package of $R$ software at the cut-off criteria of fold change (FC) $>2$ and adj. $p<0.05$. RT-qPCR assay was conducted to measure hsa_circ_0004831 expression level in CRC blood samples. A circRNA-miRNA-mRNA regulatory network of hsa_circ_0004831 was constructed based on competitive endogenous RNA mechanism and differentially expressed genes. The mRNAs co-expressed with hsa_circ_0004831 were screened at the cut-off criteria of pearson $|r|>0.3$ and $p<0.05$. Gene set enrichment analysis (GSEA) based on co-expressed mRNAs was used to explore the potential molecular function of hsa_circ_0004831.
\end{abstract}

Results: Differentially expressed circRNAs, miRNAs and mRNAs were identified and hsa_circ_0004831 had a FC value of 3.92 in CRC blood extracellular vesicles. The RT-qPCR assay showed that the hsa_circ_0004831 was up-regulated in CRC blood samples. The overall survival analysis found that high expression of hsa_circ_0004831 was linked with poorer prognosis. Finally, a circRNA-miRNA-mRNA regulatory network of hsa_circ_0004831 was constructed based on down-regulated miR-4326 and 12 up-regulated mRNAs. GSEA indicated that mRNAs co-expressed with hsa_ circ_0004831 were involved in EMT, WNT and p53 signaling pathways.

Conclusions: The study confirmed the up-regulation of hsa_circ_0004831 in CRC, and it may act as a vital prognostic biomarker. The circRNA-miRNA-mRNA regulatory network of hsa_circ_0004831 could be used to uncover the tumorigenesis and progression of CRC.

Keywords: Colorectal cancer, CircRNA, Extracellular vesicle, Competitive endogenous RNA, Prognosis

*Correspondence: fanl@sj-hospital.org

2 Department of Nursing, Shengjing Hospital of China Medical University, Shenyang, China

Full list of author information is available at the end of the article

\section{Background}

The incidence of colorectal cancer (CRC) ranks the third among all human cancers in 2018 and is a common malignant tumor in the clinic $[1,2]$. There are kinds of factors which are closely related to CRC incidence,

c) The Author(s) 2020. This article is licensed under a Creative Commons Attribution 4.0 International License, which permits use, sharing, adaptation, distribution and reproduction in any medium or format, as long as you give appropriate credit to the original author(s) and the source, provide a link to the Creative Commons licence, and indicate if changes were made. The images or other third party material in this article are included in the article's Creative Commons licence, unless indicated otherwise in a credit line to the material. If material is not included in the article's Creative Commons licence and your intended use is not permitted by statutory regulation or exceeds the permitted use, you will need to obtain permission directly from the copyright holder. To view a copy of this licence, visit http://creativeco mmons.org/licenses/by/4.0/. The Creative Commons Public Domain Dedication waiver (http://creativecommons.org/publicdomain/ zero/1.0/) applies to the data made available in this article, unless otherwise stated in a credit line to the data. 
including genetic alteration, diet, lifestyle factors of physical activity and sedentary behavior [3]. Although encouraging advancement in traditional surgery, radioand chemotherapy, the prognosis of CRC patients is very poor, especially in metastatic patients, with no more than $10 \%$ of 5-year survival rates [4]. Therefore, it is necessary to further elucidate the pathophysiological processes that occur in CRC and explore more efficient biomarkers in prognostic prediction.

As a series of novel non-coding RNAs, circular RNAs (circRNAs) are produced from precursor mRNAs backsplicing and have attracted lots of attention in science study [5]. CircRNAs were characterized by a covalently closed-loop structures without $5^{\prime}$ and $3^{\prime}$ terminals and play vital role in the processes of various cancers progression [6,7]. Extensive studies have revealed the vital importance of circRNAs in human diseases, including cancers [8-10]. Due to the circular structures being resistant to most RNA degradation, circRNAs are abundant in plasma and may present at levels ten-folds higher than those of the corresponding linear mRNA [11]. Taken together, circRNAs have the potential to be effective diagnostic and prognostic biomarkers. However, the roles of a large number of circRNAs in CRC remain unclear.

In our study, we found that hsa_circ_0004831 was upregulated in the blood of CRC patients. The KaplanMeier analysis with log-rank test found that high expression of hsa_circ_0004831 was linked with poorer prognosis. A circRNA-miRNA-mRNA regulatory network of hsa_circ_0004831 was constructed based on competitive endogenous RNA mechanism and differentially expressed genes in CRC. Moreover, GSEA showed that mRNAs co-expressed with hsa_circ_0004831 were involved in EMT, WNT and p53 signaling pathways. These findings indicated that hsa_circ_0004831 participated in important biological process and may be a potent prognostic biomarker for CRC.

\section{Methods \\ Clinical samples}

A total of 81 patients diagnosed with CRC at Shengjing Hospital of the China Medical University and 50 healthy volunteers were enrolled in present study. All the blood samples used in this research were collected with complete informed consent from the participants and ethics approval was obtained from the ethics review committee of the Shengjing Hospital of China Medical University before this study. Serum samples were extracted from blood samples after being centrifuged at $3000 \mathrm{rpm}$, $4{ }^{\circ} \mathrm{C}$ for $10 \mathrm{~min}$ and were stored at $-80{ }^{\circ} \mathrm{C}$ until RNA isolation.

\section{RNA isolation and RT-qPCR}

Blood samples in containers without coagulant were preserved at $4{ }^{\circ} \mathrm{C}$ for $4 \mathrm{~h}$ to ensure serum separation. Then, serum was stored at $-80{ }^{\circ} \mathrm{C}$ until for use after centrifuged for $10 \mathrm{~min}$ at $5000 \mathrm{rpm}$ and $3000 \mathrm{rpm}$, respectively. Total RNA was isolated from serum with TRIzol reagent (Invitrogen, Carlsbad, CA, United States) according to the manufacturer's protocol. The concentration and quality of total RNA in all samples were detected using NanoDrop ND-1000 spectrophotometer (NanoDrop, Wilmington, DE, United States). The synthesis of cDNA and RT-qPCR reactions were conducted using a reverse transcription kit and the SYBR Green kit (Takara Bio, Dalian, China). Relative gene expression was determined using the ABI 7500 System (Applied Biosystems, USA) with the following qPCR cycling program: 45 cycles including denaturation at $95{ }^{\circ} \mathrm{C}$ for $5 \mathrm{~s}$, annealing at 60 ${ }^{\circ} \mathrm{C}$ for $30 \mathrm{~s}$ and extension at $72{ }^{\circ} \mathrm{C}$ for $30 \mathrm{~s}$. GAPDH was selected as the reference gene. The primers used for qRTPCR were shown in Table 1. All genes expression levels were quantified using the $\Delta \Delta \mathrm{ct}$ method.

\section{Identification of differentially expressed circRNAs, miRNAs and mRNAs}

The circRNA and mRNA expression profiles in extracellular vesicles of CRC and normal samples were obtained from the exoRBase database [12]. The corresponding miRNA expression profile in extracellular vesicles was downloaded from the BBCancer database [13]. To ensure the reliability of data processing, the genes which have no expression value in more than half samples were excluded for analysis. The limma package [14] in $\mathrm{R}$ was used to identify differentially expressed circRNAs, miRNAs and mRNAs between CRC and normal samples. $|\log 2 \mathrm{FC}|>1$ and adj. $\mathrm{P}$ value $<0.05$ were considered to be statistically significant difference.

\section{Construction of circRNA-miRNA-mRNA network}

The miRNAs which include miRNA binding sites on hsa_circ_0004831 genome sequence were predicted with Circbank [15] database (https://www.circbank.cn). The overlaps of predicted results and down-regulated miRNAs in CRC were regarded as miRNAs which could be regulated by hsa_circ_0004831 through competitive endogenous RNA mechanism. The target genes of miRNAs were predicted by TargetScan v7.1 tool [16]. Similarly, the overlaps of predicted genes and up-regulated mRNAs in CRC were regarded as target genes which involved in competitive endogenous RNA network. Finally, the circRNA-miRNA-mRNA regulatory network was constructed and visualized using Cytoscape v3.7.1 [17]. 
Table 1 The Primers used for qRT-PCR

\begin{tabular}{|c|c|}
\hline Name & Sequence \\
\hline \multicolumn{2}{|c|}{ hsa_circ_0004831 } \\
\hline Forward & 5'- AAAGAAGAAAGAGCGTGCCG-3' \\
\hline Reverse & 5'- ATGATCATCAGAGGAGGGCG-3' \\
\hline \multicolumn{2}{|l|}{ miR-4326 } \\
\hline RT primer & 5'-GTCGTATCCAGTGCAGGGTCCGAGGTATTCGCACTGGATACGACGTCTGG-3' \\
\hline Forward & 5'-GCCCGC TGTTCCTCTGTCTCCC-3' \\
\hline Reverse & 5'-GTGCAGGGTCCGAGGT-3' \\
\hline \multicolumn{2}{|l|}{ ZBED1 } \\
\hline Forward & 5'-CCCGGACGAATTCTTCGAAATGGAGAATAAAAGCCTGGAGAG-3' \\
\hline Reverse & 5'-TGCGGATCACTAGTGCTAGCCTACAGGAAGCTGCTGTCCCTAATG-3' \\
\hline \multicolumn{2}{|l|}{ GAPDH } \\
\hline Forward & 5'-ATGGGGAAGGTGAAGGTCG-3' \\
\hline Reverse & 5'-TTACTCCTTGGAGGCCATGTG-3' \\
\hline \multicolumn{2}{|l|}{$U_{6}$} \\
\hline Forward & 5'-CTCGCTTCGGCAGCACA-3' \\
\hline Reverse & 5'-AACGCTTCACGAATTTGCGT-3' \\
\hline
\end{tabular}

\section{GSEA based on co-expressed mRNAs}

The mRNAs co-expressed with hsa_circ_0004831 in CRC and normal samples were identified using pearson correlation analysis. Pearson $|\mathrm{r}|>0.3$ and $\mathrm{p}$ value $<0.05$ were considered statistically significant. The expression matrix of co-expressed mRNAs was performed for GSEA to explore the biological differences between CRC and normal samples. The hallmark and KEGG [18] subsets in the Molecular Signatures Database (MSigDB) [19] were used as annotated gene sets during GSEA [20].

\section{Statistical analysis}

Statistical analysis used in present study was performed with GraphPad Prism v 7.00 for Windows (GraphPad Software, USA). The differences of genes expression levels between two groups were analyzed by two-tailed Student's t-test. The overall survival analysis was performed using Kaplan-Meier curves and log-rank test. The optimal cut-off threshold of low or high hsa_circ_0004831 expression was calculated by X-tile [21]. A p value of $<0.05$ was considered statistically significant.

\section{Results}

Differentially expressed circRNAs, miRNAs and mRNAs

The flowchart for this study is shown in Fig. 1. After expression profiles processing, there are $12 \mathrm{CRC}$ and 32 normal samples for circRNA and mRNA expression matrix, and $100 \mathrm{CRC}$ and 55 normal samples for miRNA expression matrix. There are totally 16,115 circRNAs, 1274 miRNAs and 16,605 mRNAs in the expression profiles used for identifying differentially expressed
RNAs. According to the cut-off criteria of FC $>2$ and adj. $\mathrm{p}<0.05$, a total of 101 differentially expressed circRNAs (28 up- and 73 down-regulated), 15 differentially expressed miRNAs ( 2 up- and 13 down-regulated) and 108 differentially expressed mRNAs (97 up- and 11 down-regulated) were identified. The detail results of differentially expression were displayed in the volcano plots and hierarchic cluster heatmaps (Fig. 2). The FC values of hsa_circ_0004831 and miR-4326 expression in CRC were 3.92 and 0.47 , respectively.

\section{hsa_circ_0004831 was up-regulated in CRC and could be} a prognostic biomarker

From circRNA expression profile, we noticed that hsa circ_0004831 expression level increased 3.92 FC on average in CRC and was significantly up-regulated compared with that in the normal samples (Fig. 3a). Similarly, the RT-qPCR assay showed that hsa_circ_0004831 was significantly up-regulated in 81 CRC blood samples compared with that in 50 healthy volunteers' samples (Fig. 3b). To investigate the clinical value of hsa_circ_000483, we performed correlation analysis between hsa_circ_0004831 expression and clinicopathological characteristics. The findings suggested that high hsa_circ_0004831 expression was significantly correlated with distant metastasis $(\mathrm{p}=0.018)$ and differentiation grade $(\mathrm{p}=0.027)$ of $\mathrm{CRC}$ (Table 2). Then, we explored the role of hsa_circ_0004831 in CRC patients prognostic prediction. The optimal cutoff threshold of low or high hsa_circ_0004831 expression calculated by X-tile was 5.1 (Fig. 3c). The overall survival analysis with Kaplan-Meier and log-rank test showed 


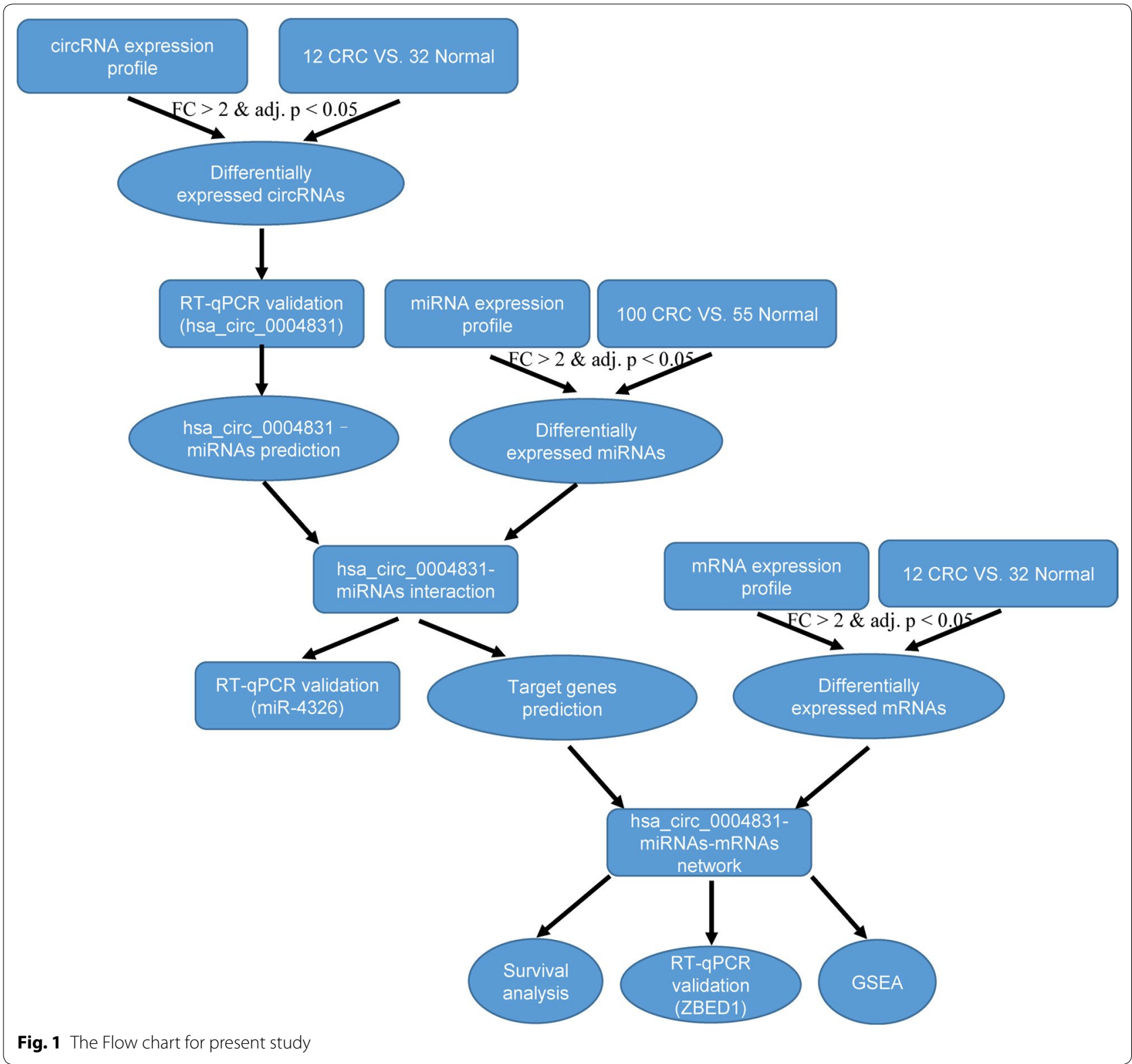

that patients with high hsa_circ_0004831 (median survival: 5.83) expression had a poorer prognosis than that with low hsa_circ_0004831 (median survival: NA) expression (Fig. 3d). Above findings indicated that hsa circ_0004831 may be a potent prognostic biomarker for CRC.

\section{The circRNA-miRNA-mRNA regulatory network of hsa circ 0004831}

Firstly, we obtained 22 miRNAs which include miRNA binding sites on hsa_circ_0004831 genome sequence from Circbank database. After integrating with 13 down-regulated miRNAs, hsa-miR-4326 was identified to be regulated by hsa_circ_0004831 through competitive endogenous RNA mechanism (Fig. 4a). The miRNA expression profile showed that hsa-miR-4326 was significantly down-regulated in CRC compared with that in the normal samples. Besides, RT-qPCR assay confirmed that miR-4326 was down-regulated in 81 CRC blood samples compared with that in 50 healthy volunteers' samples (Fig. 4b). Then, we predicted 3629 target genes of hsa-miR-4326 from TargetScan. Using similar methods, a total of 12 mRNAs were identified as target genes which involved in competitive endogenous RNA network (Fig. 4c). Thus, a circRNA-miRNA-mRNA regulatory network involved in hsa_circ_0004831, hsa-miR-4326 


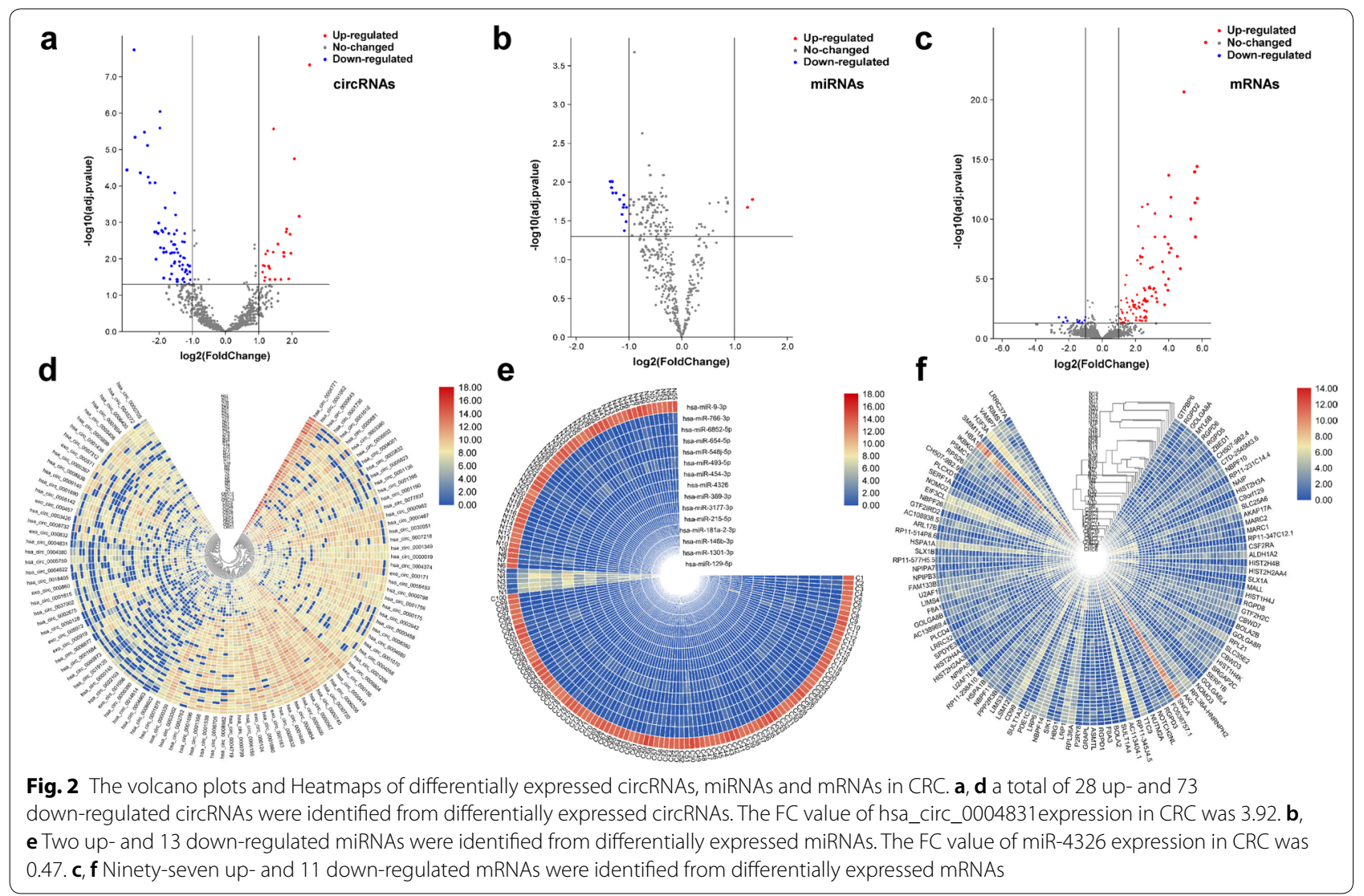

and 12 mRNAs was visualized using Cytoscape 3.7.1 (Fig. 4d).

\section{GSEA based on co-expressed mRNAs}

In order to further study the functional pathways which hsa_circ_0004831 may participate in, we first obtained mRNAs co-expressed with hsa_circ_0004831 in CRC and normal samples using pearson correlation analysis. At the conditions of pearson $|\mathrm{r}|>0.3$ and $\mathrm{p}$ value $<0.05$, a total of 2503 co-expressed mRNAs were identified. GSEA based on the expression matrix of co-expressed mRNAs showed that hsa_circ_0004831 were involved in EMT, WNT and p53 signaling pathways (Fig. 5a). Furthermore, we visualized the pearson expression correlation of hsa_circ_0004831 and 12 target mRNAs. The results showed that the expression of hsa_circ_0004831 was positively correlated with that of target mRNAs (Fig. $5 b$ ). The mRNA expression profile indicated that 12 target mRNAs were all significantly up-regulated in CRC compared with that in the normal samples (Fig. $5 \mathrm{c}$ ). Meanwhile, the mRNA expression level of ZBED1 was detected by RT-qPCR assay. Compared with 50 healthy volunteers' samples, ZBED1 was found to be up-regulated in 81 CRC blood samples (Fig. 5d).

\section{Discussion}

Mounting studies showed that circRNAs are closely related to CRC carcinogenesis [22-24]. The circRNAmiRNA-mRNA competitive endogenous RNA network plays a critical role in CRC incidence, providing novel insights into the pathogenesis of CRC. The present study identified differentially expressed circRNAs, miRNAs and mRNAs between CRC and normal blood samples. We found that hsa_circ_0004831 expression level increased $3.92 \mathrm{FC}$ on average in CRC. However, the role of hsa_circ_0004831 in CRC are remain unclear. The RT-qPCR assay also confirmed that hsa_circ_0004831 was significantly up-regulated in 81 CRC blood samples compared with that in 50 healthy volunteers' samples. All these results revealed that hsa_circ_0004831 may act as an oncogene in CRC.

Hsa_circ_0004831 is a transcription product of the remodeling and spacing factor 1 (RSF1) gene located on chromosome 11. The potential function of hsa circ_0004831 in the tumorigenesis and progression of CRC attracted our interest. The Kaplan-Meier analysis with log-rank test found that high expression of hsa_circ_0004831 was linked with poorer prognosis. Due to the stable existence of circRNAs in plasma and 


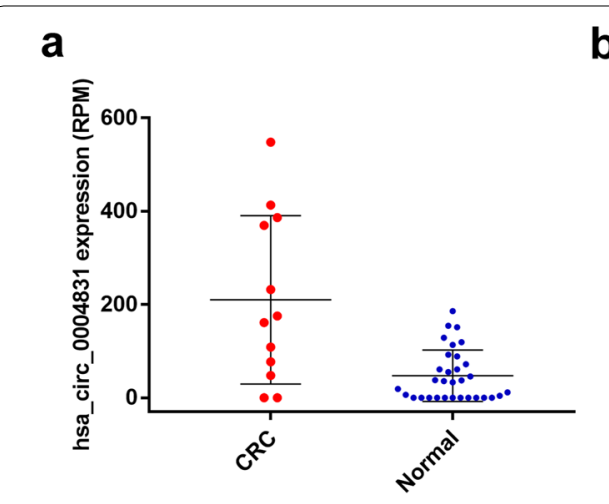

C

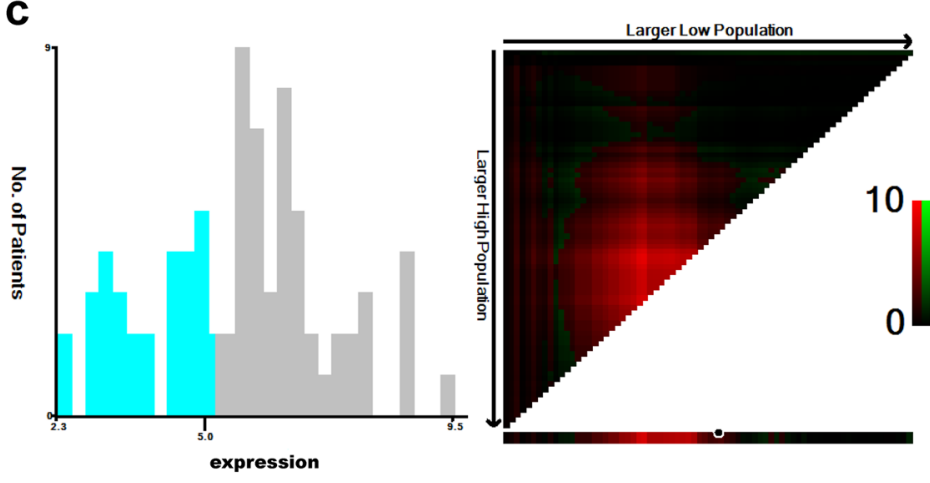

b

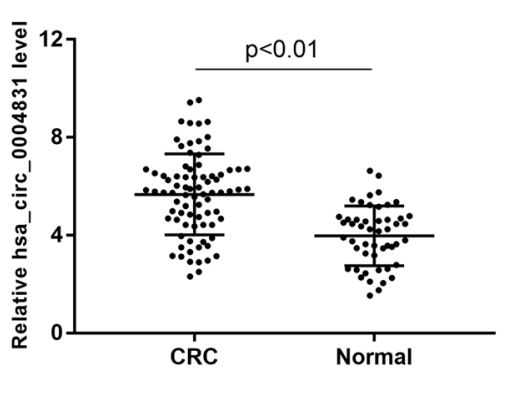

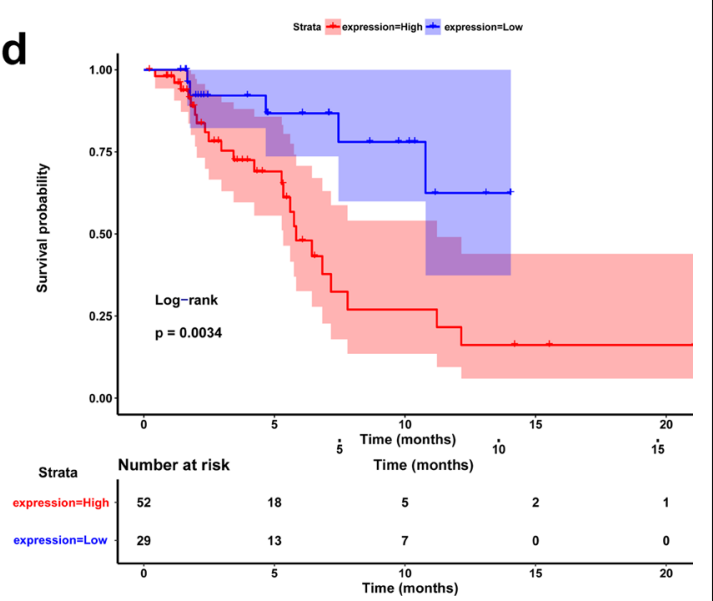

Fig. 3 The expression of hsa_circ_0004831 in CRC and survival analysis. a Hsa_circ_0004831 was up-regulated in CRC (exoRBase data). b Hsa_ circ_0004831 was up-regulated in CRC (RT-qPCR data). c The optimal cut-off threshold of low or high hsa_circ_0004831 expression calculated by $X$-tile was 5.1. d High hsa_circ_0004831 expression group had a poorer prognosis compared with the low expression group $(p=0.0034)$

favorable patient compliance, hsa_circ_0004831 may be a potent prognostic biomarker for CRC.

Present evidences showed that circRNAs can act as competitive endogenous RNAs to reduce the miRNAs expression levels and release their targeted inhibition to mRNAs, thereby mediating the expression of proteincoding genes [25-27]. Lin et al. found that circCYFIP2 may act as an oncogenic circRNA to promote gastric cancer progression through miR-1205/E2F1 axis and could be a potent therapeutic target for gastric cancer [28]. Hsa_circ_0006168 was found to be significantly increased in the tissue and cell line level of esophageal squamous cell cancer and may regulate the expression of mTOR via sponging miRNA-100 [29]. Besides, $\mathrm{Su}$ et al. found that hsa_circ_0070269 can play a vital role in hepatocellular carcinoma progression through sponging miR-182 and thus increasing NPTX1 expression [30]. However, the competitive endogenous RNA mechanism of hsa_circ_0004831 in CRC is still unclear. Thus, we constructed a circRNA-miRNA-mRNA regulatory network of hsa_circ_0004831 based on competitive endogenous RNA mechanism and differentially expressed genes in CRC. The findings can provide novel insights into the pathogenesis of CRC.

To further investigate the biological pathways which hsa_circ_0004831 may participate in, we performed GSEA using the expression matrix of mRNAs coexpressed with hsa_circ_0004831 in CRC and normal samples. The findings of GSEA showed that hsa_circ_0004831 can participate in EMT, WNT and p53 signaling pathways. EMT is a common biological pathway that is necessary for cancer progression since it converts immobile epithelial cells into active mesenchymal cells and thereby promotes metastasis [31]. For example, a previous study demonstrated that silencing circ_0001946 can suppress CRC cell growth, migration, and invasion through EMT pathway inhibition [32]. Ma et al. found that circ5615 may function as competing endogenous RNA of miR-149-5p and activate Wnt/ $\beta$-catenin pathway to promote CRC progression [33]. Moreover, Li et al. first identified that circRNA CBL.11/miR-6778-5p/YWHAE axis together with the p53 pathway can regulate apoptosis and proliferation of CRC cells [34]. To the best of our knowledge, the role of hsa_circ_0004831 associated 
Table 2 Correlation between hsa_circ_0004831 expression and clinicopathological characteristics

\begin{tabular}{|c|c|c|c|c|}
\hline Characteristics & $\begin{array}{l}\text { No. } \\
\text { of patients }\end{array}$ & $\begin{array}{l}\text { hsa } \\
\text { circ_0004831 } \\
\text { (M } \pm \text { SEM) }\end{array}$ & t value & $p$ \\
\hline \multicolumn{5}{|l|}{ Age (years) } \\
\hline$\geq 50$ & 45 & $5.59 \pm 0.27$ & 0.37 & 0.715 \\
\hline$<50$ & 36 & $5.73 \pm 0.25$ & & \\
\hline \multicolumn{5}{|l|}{ Gender } \\
\hline Male & 47 & $5.50 \pm 0.23$ & 1.06 & 0.291 \\
\hline Female & 34 & $5.89 \pm 0.29$ & & \\
\hline \multicolumn{5}{|c|}{ Distant metastasis } \\
\hline MO & 36 & $5.18 \pm 0.28$ & 2.43 & $0.018^{*}$ \\
\hline M1 & 45 & $6.06 \pm 0.23$ & & \\
\hline \multicolumn{5}{|l|}{ TNM stage } \\
\hline $1+\|$ & 33 & $5.75 \pm 0.26$ & 0.36 & 0.718 \\
\hline$I I I+I V$ & 48 & $5.61 \pm 0.25$ & & \\
\hline \multicolumn{5}{|c|}{ Differentiation grade } \\
\hline Well/moderate & 42 & $5.28 \pm 0.24$ & 2.26 & $0.027^{*}$ \\
\hline Poor & 39 & $6.09 \pm 0.26$ & & \\
\hline \multicolumn{5}{|c|}{ Lymph node metastasis } \\
\hline Negative & 35 & $5.70 \pm 0.27$ & 0.15 & 0.880 \\
\hline Positive & 46 & $5.64 \pm 0.25$ & & \\
\hline
\end{tabular}

with EMT, WNT and p53 signaling pathways in CRC was undefined. Above all, the present study suggested that hsa_circ_0004831 participated in vital cancer-related biological process in CRC and provided novel target into the treatment of CRC.

However, there were also several drawbacks in present study. The study design only confirmed genes expression level and was short of findings from in vivo/vitro experiments. Even so, the subject matter of present study can be of interest to researchers in the field, and we will conduct in-depth study in further explorations. Besides, we selected TRIZOL reagent to extract RNA from serum samples, which may be not an optimal protocol. At least, the concentration and quality of total RNA in all samples were guaranteed, and we will also improve the relevant protocols in future study.

\section{Conclusions}

This study identified differentially expressed circRNAs, miRNAs and mRNAs in CRC, and RT-qPCR confirmed the up-regulation of hsa_circ_0004831 in CRC. The Kaplan-Meier analysis with log-rank test found that high expression of hsa_circ_0004831 was linked with poorer prognosis. The correlation analysis between

${ }^{*} p<0.05$ was considered statistically significant

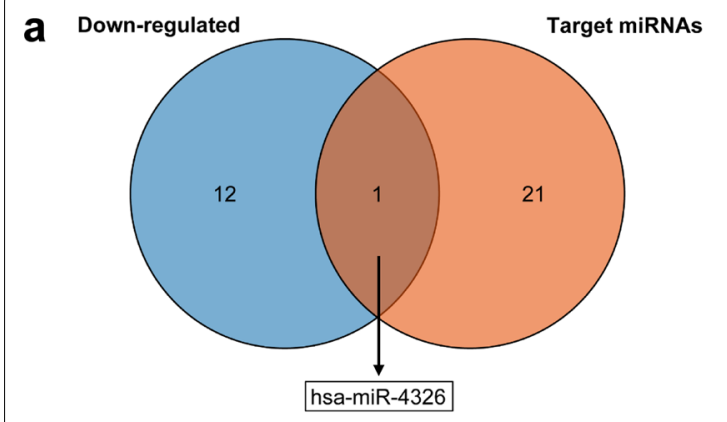

C Up-regulated

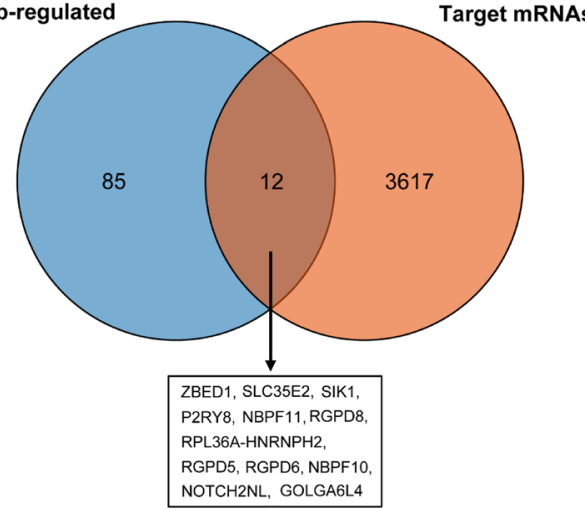

b
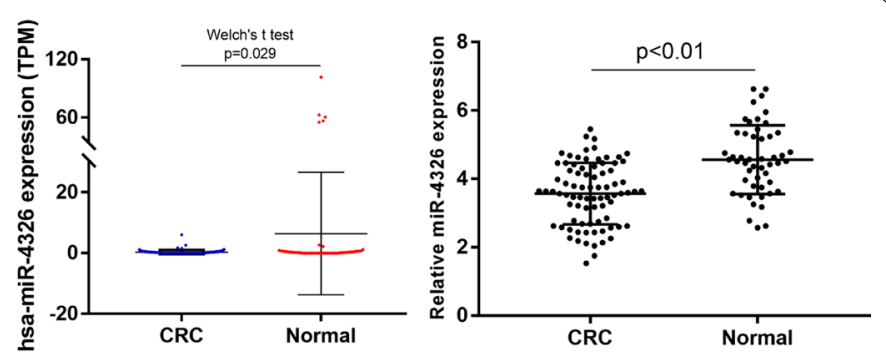

d

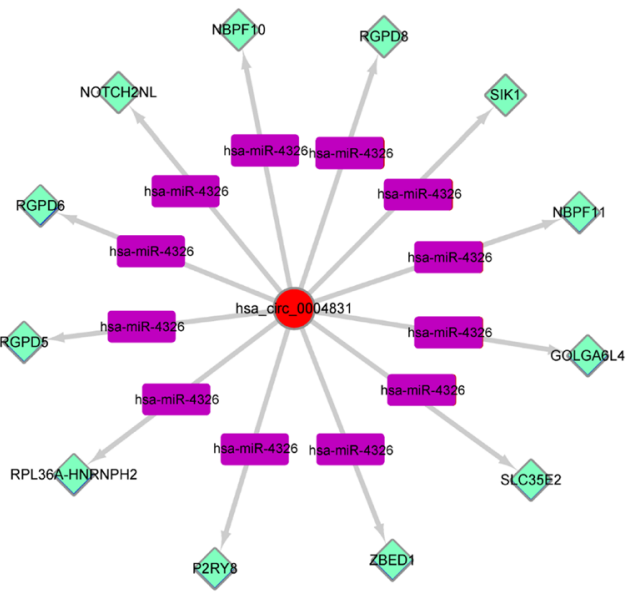

Fig. 4 The circRNA-miRNA-mRNA regulatory network construction of hsa_circ_0004831. a One miRNA (hsa-miR-4326) was identified to be regulated by hsa_circ_0004831 through competitive endogenous RNA mechanism. b Hsa-miR-4326 was down-regulated in CRC (exoRBase data and RT-qPCR assay). c Twelve mRNAs were identified as target genes which involved in competitive endogenous RNA network. $\mathbf{d}$ CircRNA-miRNA-mRNA regulatory network of hsa_circ_0004831 in CRC 

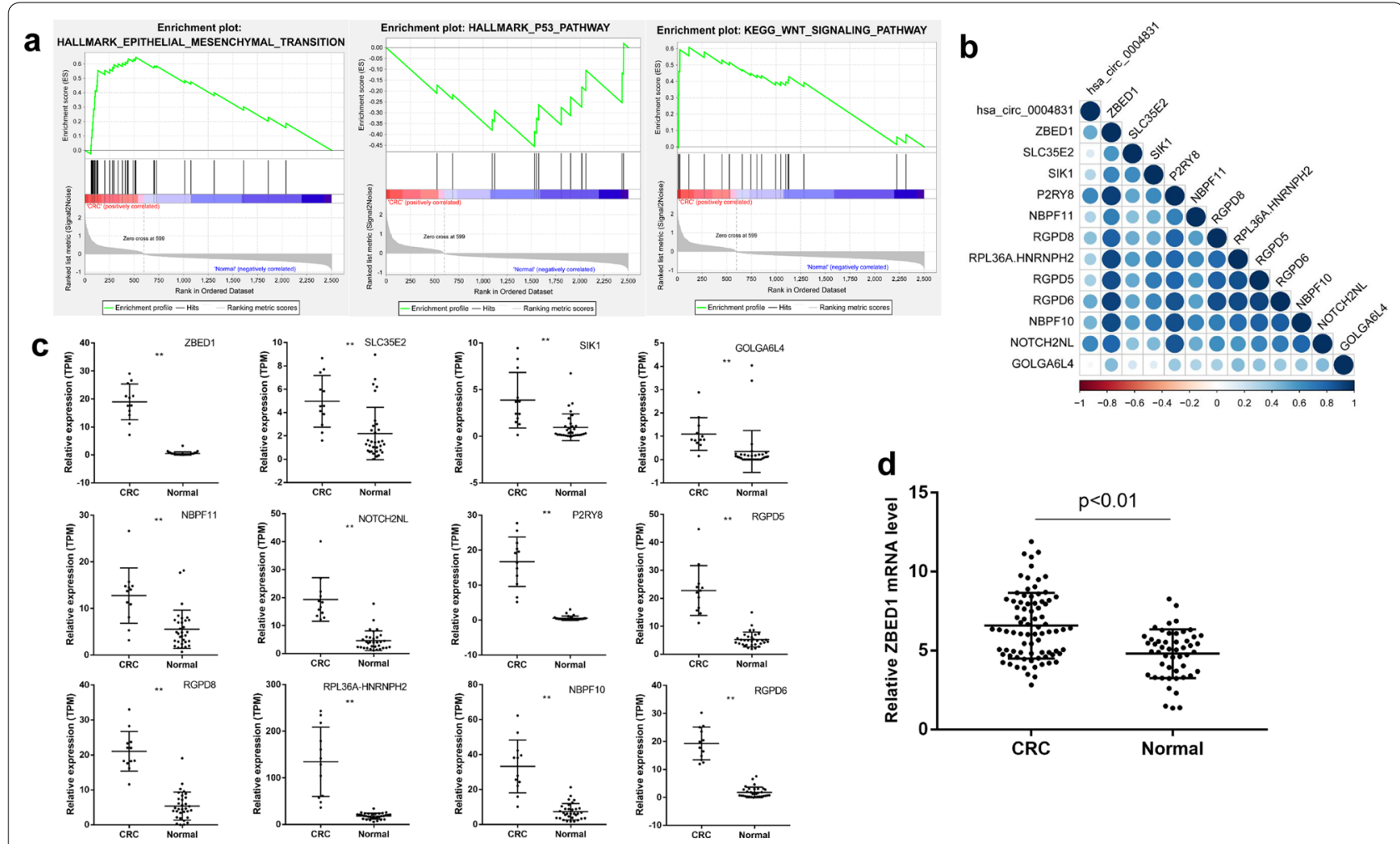

Fig. 5 GSEA and identification of mRNAs co-expressed with hsa_circ_0004831. a GSEA based on the expression matrix of co-expressed mRNAs showed that hsa_circ_0004831 were involved in EMT, WNT and p53 signaling pathways. b pearson expression correlation showed that the expression of hsa_circ_0004831 was positively correlated with that of 12 target mRNAs. c The distribution plots of 12 target mRNAs expression in CRC and the normal samples from mRNA expression profiles. $\mathbf{d}$ RT-qPCR assay indicated that ZBED1 expression was up-regulated in 81 CRC blood samples

hsa_circ_0004831 expression and clinicopathological characteristics suggested that high hsa_circ_0004831 expression was significantly correlated with distant metastasis $(\mathrm{p}=0.018)$ and differentiation grade $(\mathrm{p}=0.027)$. A circRNA-miRNA-mRNA regulatory network of hsa_circ_0004831 was constructed based on competitive endogenous RNA mechanism and differentially expressed genes in CRC. Moreover, GSEA showed that mRNAs co-expressed with hsa_circ_0004831 were involved in EMT, WNT and p53 signaling pathways. These findings indicated that hsa_circ_0004831 participated in important biological process and may be a potent prognostic biomarker for CRC.

\section{Abbreviations}

CRC: Colorectal cancer; FC: Fold change; RT-qPCR: Real time-quantitative polymerase chain reaction; GSEA: Gene set enrichment analysis; EMT: Epithelialmesenchymal transition; CircRNAs: Circular RNAs; KEGG: Kyoto Encyclopaedia of Genes and Genomes; RPM: Reads per million mapped reads; NA: Not available; Adj: Adjusted.

\section{Acknowledgements}

Not applicable.

\section{Authors' contributions}

Xing LL and Fan L conceived this study; Xing LL, Xia MY and Jiao X performed the experiments, and Xing LL was a major contributor in writing the manuscript. All authors read and approved the final manuscript.

\section{Funding \\ None.}

\section{Availability of data and materials}

The datasets used and/or analysed during the current study are available from the corresponding author on reasonable request.

\section{Ethics approval and consent to participate}

Informed consent forms have been signed by all patients before this study. The research protocol has been approved by the Ethics Committee of the Shengjing Hospital of China Medical University and is based on the ethical principles of medical research involving human subjects in the Helsinki Declaration (No.:2018PS362K).

\section{Consent for publication}

Not applicable.

\section{Competing interests}

The authors declare that they have no competing interests.

\section{Author details}

${ }^{1}$ Department of Radiology, Shengjing Hospital of China Medical University, Shenyang, China. ${ }^{2}$ Department of Nursing, Shengjing Hospital of China Medical University, Shenyang, China. 
Received: 6 August 2020 Accepted: 9 November 2020

Published online: 19 November 2020

\section{References}

1. Dekker E, Tanis PJ, Vleugels JLA, Kasi PM, Wallace MB. Colorectal cancer. Lancet. 2019;394(10207):1467-80.

2. Bray F, Ferlay J, Soerjomataram I, Siegel RL, Torre LA, Jemal A. Global cancer statistics 2018: GLOBOCAN estimates of incidence and mortality worldwide for 36 cancers in 185 countries. CA Cancer J Clin. 2018;68(6):394-424.

3. Kerr J, Anderson C, Lippman SM. Physical activity, sedentary behaviour, diet, and cancer: an update and emerging new evidence. Lancet Oncol. 2017;18(8):e457-71.

4. Haggar FA, Boushey RP. Colorectal cancer epidemiology: incidence, mortality, survival, and risk factors. Clin Colon Rectal Surg. 2009;22(4):191-7.

5. Kristensen LS, Andersen MS, Stagsted LVW, Ebbesen KK, Hansen TB, Kjems J. The biogenesis, biology and characterization of circular RNAs. Nat Rev Genet. 2019;20(11):675-91.

6. Kristensen LS, Hansen TB, Veno MT, Kjems J. Circular RNAs in cancer: opportunities and challenges in the field. Oncogene. 2018;37(5):555-65.

7. Li HM, Ma XL, Li HG. Intriguing circles: Conflicts and controversies in circular RNA research. Wiley Interdiscip Rev RNA. 2019;10(5):e1538.

8. Fang G, Ye BL, Hu BR, Ruan XJ, Shi YX. CircRNA_100290 promotes colorectal cancer progression through miR-516b-induced downregulation of FZD4 expression and Wnt/beta-catenin signaling. Biochem Biophys Res Commun. 2018;504(1):184-9.

9. Xu G, Chen Y, Fu M, Zang X, Cang M, Niu Y, Zhang W, Zhang Y, Mao Z, Shao M, et al. Circular RNA CCDC66 promotes gastric cancer progression by regulating c-Myc and TGF-beta signaling pathways. J Cancer. 2020;11(10):2759-68.

10. Chen B, Huang S. Circular RNA: An emerging non-coding RNA as a regulator and biomarker in cancer. Cancer Lett. 2018;418:41-50.

11. Zhao ZJ, Shen J. Circular RNA participates in the carcinogenesis and the malignant behavior of cancer. RNA Biol. 2017;14(5):514-21.

12. Li S, Li Y, Chen B, Zhao J, Yu S, Tang Y, Zheng Q, Li Y, Wang P, He X, et al. exoRBase: a database of circRNA, IncRNA and mRNA in human blood exosomes. J Nucleic Acids Res. 2018;46(D1):D106-12.

13. Zuo Z, Hu H, Xu Q, Luo X, Peng D, Zhu K, Zhao Q, Xie Y, Ren J. BBCancer: an expression atlas of blood-based biomarkers in the early diagnosis of cancers. Nucleic Acids Res. 2020;48(D1):D789-96.

14. Ritchie ME, Phipson B, Wu D, Hu Y, Law CW, Shi W, Smyth GK. limma powers differential expression analyses for RNA-sequencing and microarray studies. Nucleic Acids Res. 2015;43(7):e47.

15. Liu M, Wang Q, Shen J, Yang BB, Ding X. Circbank: a comprehensive database for circRNA with standard nomenclature. RNA Biol. 2019;16(7):899-905.

16. Agarwal V, Bell GW, Nam JW, Bartel DP. Predicting effective microRNA target sites in mammalian mRNAs. Elife. 2015;4:e05005.

17. Shannon P, Markiel A, Ozier O, Baliga NS, Wang JT, Ramage D, Amin N, Schwikowski B, Ideker T. Cytoscape: a software environment for integrated models of biomolecular interaction networks. Genome Res. 2003;13(11):2498-504.

18. Kanehisa M, Sato Y, Kawashima M, Furumichi M, Tanabe M. KEGG as a reference resource for gene and protein annotation. Nucleic Acids Res. 2016;44(D1):D457-62.
19. Liberzon A, Birger C, Thorvaldsdottir H, Ghandi M, Mesirov JP, Tamayo P. The Molecular Signatures Database (MSigDB) hallmark gene set collection. Cell Syst. 2015;1(6):417-25

20. Subramanian A, Tamayo P, Mootha VK, Mukherjee S, Ebert BL, Gillette MA, Paulovich A, Pomeroy SL, Golub TR, Lander ES, et al. Gene set enrichment analysis: a knowledge-based approach for interpreting genome-wide expression profiles. Proc Natl Acad Sci USA. 2005;102(43):15545-50.

21. Camp RL, Dolled-Filhart M, Rimm DL. X-tile: a new bio-informatics tool for biomarker assessment and outcome-based cut-point optimization. Clin Cancer Res. 2004;10(21):7252-9.

22. Li XN, Wang ZJ, Ye CX, Zhao BC, Huang XX, Yang L. Circular RNA circVAPA is up-regulated and exerts oncogenic properties by sponging miR-101 in colorectal cancer. Biomed Pharmacother. 2019;112:108611.

23. Zhu CL, Sha X, Wang Y, Li J, Zhang MY, Guo ZY, Sun SA, He JD. Circular RNA hsa_circ_0007142 Is Upregulated and Targets miR-103a-2-5p in Colorectal Cancer. J Oncol. 2019;2019:9836819.

24. Geng Y, Zheng X, Hu W, Wang Q, Xu Y, He W, Wu C, Zhu D, Wu C, Jiang J. Hsa_circ_0009361 acts as the sponge of miR-582 to suppress colorectal cancer progression by regulating APC2 expression. Clin Sci (Lond). 2019;133(10):1197-213.

25. Karreth FA, Pandolfi PP. ceRNA cross-talk in cancer: when ce-bling rivalries go awry. Cancer Discov. 2013;3(10):1113-21.

26. Qi X, Zhang DH, Wu N, Xiao JH, Wang X, Ma W. ceRNA in cancer: possible functions and clinical implications. J Med Genet. 2015;52(10):710-8.

27. Thomson DW, Dinger ME. Endogenous microRNA sponges: evidence and controversy. Nat Rev Genet. 2016;17(5):272-83.

28. Lin J, Liao S, Li E, Liu Z, Zheng R, Wu X, Zeng W. circCYFIP2 Acts as a Sponge of miR-1205 and Affects the Expression of Its Target Gene E2F1 to Regulate Gastric Cancer Metastasis. Mol Ther Nucleic Acids. 2020;21:121-32.

29. Shi Y, Guo Z, Fang N, Jiang W, Fan Y, He Y, Ma Z, Chen Y. hsa_circ_0006168 sponges miR-100 and regulates mTOR to promote the proliferation, migration and invasion of esophageal squamous cell carcinoma. Biomed Pharmacother. 2019;117:109151.

30. Su X, Su J, He H, Zhan Y, Liu H. Hsa_circ_0070269 inhibits hepatocellular carcinoma progression through modulating miR-182/NPTX1 axis. Biomed Pharmacother. 2019;120:109497.

31. Yang J, Weinberg RA. Epithelial-mesenchymal transition: at the crossroads of development and tumor metastasis. Dev Cell. 2008;14(6):818-29.

32. Deng Z, Li X, Wang H, Geng Y, Cai Y, Tang Y, Wang Y, Yu X, Li L, Li R. Dysregulation of CircRNA_0001946 contributes to the proliferation and metastasis of colorectal cancer cells by targeting microRNA-135a-5. Front Genet. 2020;11:357.

33. Ma Z, Han C, Xia W, Wang S, Li X, Fang P, Yin R, Xu L, Yang L. circ5615 functions as a ceRNA to promote colorectal cancer progression by upregulating TNKS. Cell Death Dis. 2020;11(5):356.

34. Li H, Jin X, Liu B, Zhang P, Chen W, Li Q. CircRNA CBL.11 suppresses cell proliferation by sponging miR-6778-5p in colorectal cancer. BMC Cancer. 2019;19(1):826

\section{Publisher's Note}

Springer Nature remains neutral with regard to jurisdictional claims in published maps and institutional affiliations.

Ready to submit your research? Choose BMC and benefit from:

- fast, convenient online submission

- thorough peer review by experienced researchers in your field

- rapid publication on acceptance

- support for research data, including large and complex data types

- gold Open Access which fosters wider collaboration and increased citations

- maximum visibility for your research: over $100 \mathrm{M}$ website views per year

At BMC, research is always in progress.

Learn more biomedcentral.com/submissions 